\title{
Probing the band structure of hydrogen-free amorphous carbon and the effect of nitrogen incorporation
}

\author{
Y. Miyajima ${ }^{1}$, Y. Tison ${ }^{1}$, C. E. Giusca ${ }^{1}$, V. Stolojan ${ }^{1}$, H. Watanabe ${ }^{2}$, H. Habuchi ${ }^{2}$, \\ S.J. Henley ${ }^{1}$, J.M. Shannon ${ }^{1}$ and S.R.P. Silva ${ }^{1 *}$
}

\author{
${ }^{1}$ Nano-Electronics Centre, Advanced Technology Institute, University of Surrey, \\ Guildford, GU2 7XH, UK
}

\author{
${ }^{2}$ Electrical and Computer Engineering, Gifu National College of Technology, \\ Kamimakuwa, Motosu, Gifu, 501-0495, Japan
}

\begin{abstract}
Amorphous carbon and carbon nitride bottom gate thin film transistors have been fabricated, which show bulk carrier field effect mobilities around $10^{-3}\left(\mathrm{~cm}^{2} \mathrm{~V}^{-}\right.$ ${ }^{1} \mathrm{~s}^{-1}$ ) which is orders higher than the previously reported values with p-channel devices at high electric fields between source and drain. The incorporation of nitrogen atoms into the amorphous carbon films deposited by pulsed laser deposition was studied using a wide range of techniques in order to understand the role of nitrogen in the conduction mechanism at high fields. The density of the states (DOS) was measured with several techniques such as electron energy loss spectroscopy, scanning tunnelling spectroscopy, and ultraviolet photoelectron

* Corresponding author. E-mail address: S. Si Iva@surrey. ac. uk (S. R. P. Si Iva)
\end{abstract}


spectroscopy, whereas the joint density of states (JDOS), corresponding to the transitions of electrons from the valence to the conduction bands, were obtained by optical transmittance and photothermal deflection spectroscopy. These measurements when combined can provide unparallel data on the shape and magnitude of the energy band states which are crucial to the understanding of the materials properties and thus opto-electronic applications for these thin films. In this report, the conduction mechanism will be discussed with a band diagram drawn based on the experimentally obtained DOS and JDOS measurements.

\section{INTRODUCTION}

Amorphous carbon (a-C) is an interesting material as it can contain three types of hybridised carbon atoms: $\mathrm{sp}, \mathrm{sp}^{2}$ and $\mathrm{sp}^{3} \cdot$ [1-3] This unique property has recently been used to prepare superlattices consisting of different fractions of $\mathrm{sp}^{3}$ bonded carbon layers, prepared using pulsed laser deposition (PLD), and has led to the demonstration of resonant tunnelling diodes, which is the first step towards large area high speed electronics.[4] Another remarkable property of a-C is its smooth surface which allows very precise thickness control with sub-nanometer accuracy.[5] Most recently, these materials are being examined for low $\mathrm{k}$ dielectrics, $[6,7]$ which could form the backbone of a future material used in all integrated circuits owing to its unique electronic and mechanical properties.

According to ab-initio calculations and first principle simulations, [8] a-C is naturally slightly p-type due to the presence of defects. Here, defects are classified to be two types; $\mathrm{sp}^{3}$ or $\mathrm{sp}^{2}$ dangling bonds and any $\mathrm{sp} 2$ cluster containing an odd number of $\mathrm{sp}^{2}$ sites $[8,9]$ It also indicates that the $\pi$ and $\pi^{*}$ bands are localized, whereas the $\sigma$ and $\sigma^{*}$ band are delocalized. The carrier type of a-C was confirmed 
by scanning tunnelling spectroscopy (STS) measurements which provide a spectrum of the local density of states (LDOS) close to the Fermi level. The valence band structure of a-C was analysed using ultraviolet photoelectron spectroscopy (UPS), and has been shown to consist of $\sigma$ and $\pi$ bands. [10-13]

Silva et al. reported the effect of n-type doping through nitrogen incorporation in a-C:H films.[14] Arena et al. showed that STM measurements on amorphous carbon nitride also indicate the shoulder of the conduction band becomes steeper when nitrogen is incorporated, which is an effect of n-type doping.[15, 16] In addition to the $\pi$ and $\sigma$ bands of carbon, the UPS spectra of non-hydrogenated [17] and hydrogenated amorphous carbon nitride [18] $\left(\mathrm{a}-\mathrm{CN}_{\mathrm{x}}, \mathrm{a}-\mathrm{CN}_{\mathrm{x}}: \mathrm{H}\right)$ exhibit peaks attributed to graphitic $\mathrm{C}-\mathrm{N}$ and the nitrogen lone pairs, which can be seen in the $\beta$ $\mathrm{C}_{3} \mathrm{~N}_{4}$ like structure. The presence of the $\mathrm{N}$ lone pair was also confirmed by direct current-constant photocurrent measurement (dc-CPM) and photoconductivity measurements, assuming the transition from this $\mathrm{N}$ lone pair is to the empty $\pi^{*}$ band.[19] G. Frachini et al. also reported the role of nitrogen lone pair to the optical properties in $\mathrm{a}-\mathrm{CN}_{\mathrm{x}}: \mathrm{H}$ using spectroscopic ellipsometry.[20] The bnding in amorphous carbon nitride was summarized elsewhere.[21, 22]

There have been several reports on a-C/a-CN $\mathrm{CN}_{\mathrm{x}}$ thin film transistors (TFTs), where mobility less than $10^{-3} \mathrm{~cm}^{2} \mathrm{~V}^{-1} \mathrm{~s}^{-1}$ has been measured, with carrier types of $\mathrm{p}$ and n-type for a-C/a-CN $\mathrm{CN}_{\mathrm{x}}$, respectively. The applied electric fields between source and drain are with relatively low electric field region, and therefore the conduction mechanism was attributed to conduction through localized states (via hopping), much like those found in conjugated polymers.[23-25] 
It was also reported that both the a-C and $a-\mathrm{CN}_{\mathrm{x}}$ thin film transistor (TFT) show p-type conduction through a band close to the edge of the extended states ( $\sigma$ band) with their mobility around $10^{-3} \mathrm{~cm}^{2} \mathrm{~V}^{-1} \mathrm{~S}^{-1}$ at high fields between source and drain.[26] This value is within those predicted on the Poole-Frenkel model.[27-29]

The difference in the carrier types between previous reports on $\mathrm{a}-\mathrm{CN}_{\mathrm{x}}$ and that presented in this study was explained by the different conduction mechanism attributed to the charge conduction which was heavily influenced by the band structure. This is based on the fact that nitrogen modifications to the band structure occur mainly close to the Fermi level. Thus, the extended states are not largely affected by the nitrogen incorporation, and thus, the a- $\mathrm{CN}_{\mathrm{x}}$ may be considered as a p-type semiconductor at high fields. However, there are no reports which show the change in the carrier conduction which is then correlated to a measured whole band structure. Due to most of the previous reports using limited numbers of measurement techniques for the different samples, which were anyways deposited by different techniques, no complete view on the band structure could be proposed. This we believe is the first time such an experiment can be conducted fully.

Electron energy loss spectroscopy (EELS) can provide information on the empty conduction band from the carbon $\mathrm{K}$ edge, as it corresponds to the transition from a 1s core orbital to unoccupied conduction states.[30] Here, when the 1s state is considered as having a narrow energy width, much akin to a delta function, the EELS carbon $\mathrm{K}$ edge can be treated as the shape of the empty conduction band. Ultraviolet photoelectron spectroscopy (UPS) detects the photoelectrons emitted from the occupied valence band due to the absorbed ultraviolet. Scanning tunneling 
spectroscopy (STS) can provide the shape of the DOS close to the Fermi level via tunneling between the tip and the biased sample surface.

Therefore, it is possible to measure the entire DOS/band structure, if one were to perform the following three types of measurements on a material: EELS, UPS and STS. There are two main differences between these techniques. EELS data gives bulk information since the spectra are obtained using transmitted electrons through samples. Whereas, UPS and STS are purely surface analysis techniques. Secondly, EELS and UPS obtain the information from relatively large areas compared with STS, since STS detects tunnelling between an atomically sharp tip and the surface of the samples, therefore, the DOS evaluated by STS may be different from the DOS evaluated using other techniques.[31] Even some techniques are surface sensitive techniques, it is important to actually detect the sharp of DOS.

In order to investigate the band structure more accurately, the joint density of states (JDOS) is an important point to discuss. The JDOS arises from the transition of electrons from the valence band to the conduction band, and therefore, the changes in the valence and the conduction bands is reflected in this convoluted signal.[32] Thus JDOS is important from the optical properties and application point of views, in addition to the electronic understanding of this material. The optical transmittance and photothermal deflection spectroscopy (PDS) were measured in order to investigate the JDOS, and provide further confirmation as to the shape and magnitude of the bands measured. Urbach energy is one of the important parameters for amorphous semiconductors, but it is difficult to the detect 
band tail using conventional optical transmittance. This is why the PDS is measured additionally.[33, 34]

Figure 1 shows the principle of the different measurements in obtaining the DOS ((1) EELS, (2) STS and (3) UPS) and JDOS ((4) optical transmittance and (5) PDS) for our films. Thus, when these measurements are carefully considered and integrated into a single framework, the whole picture of the band structure is more clearly accessible. Once the whole electronic structure is understood, this helps our understanding the electrical conduction mechanism much deeper.
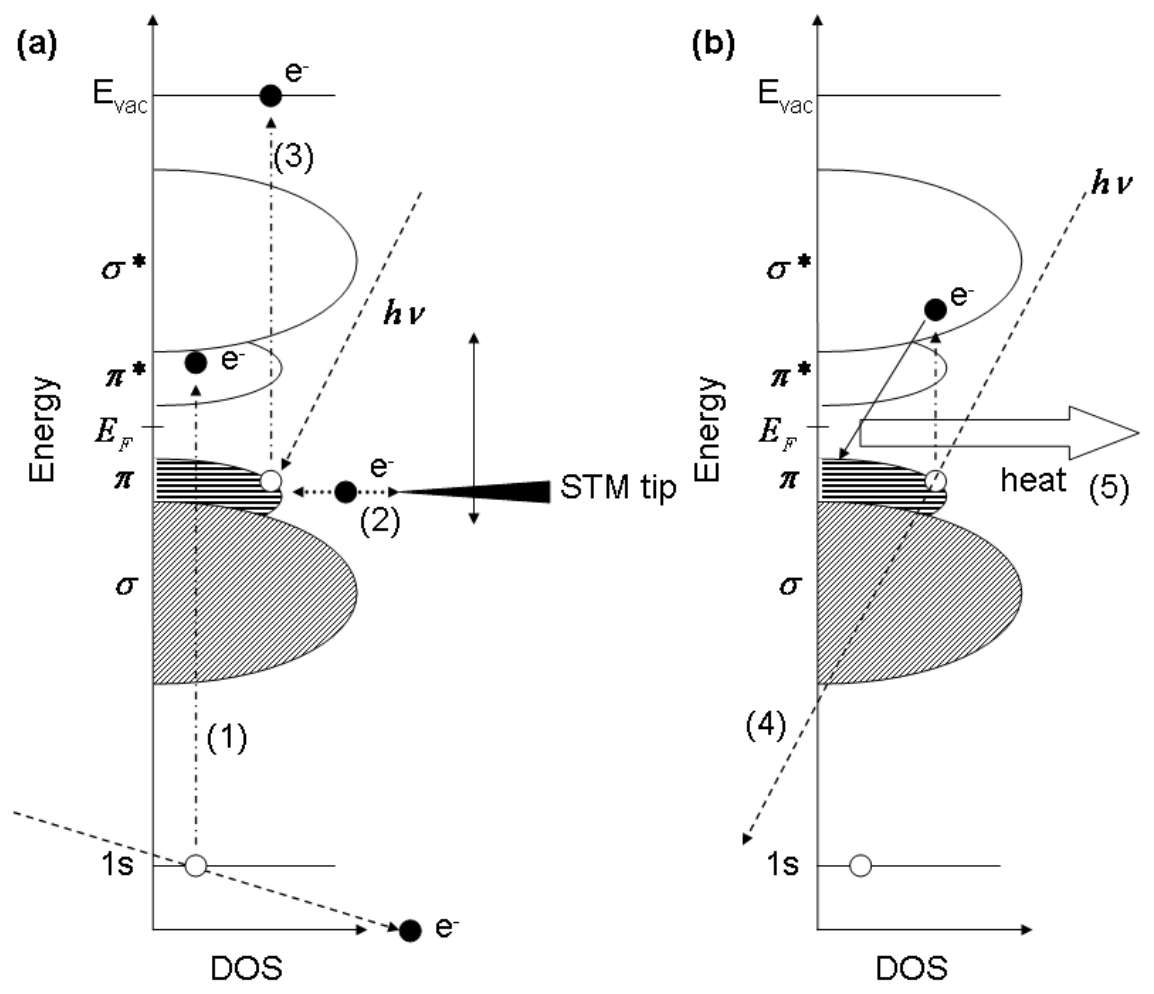

Figure 1: Schematic diagram of the measurement principles of: (a) Measurement of the density of states and (b) The joint density of states. Measurement techniques shown include: (1) EELS, (2) STS, (3) UPS, (4) optical transmittance, and, (5) PDS. 
Furthermore, three terminal measurements can identify the carrier type involved in the conduction process. All this information can then allow one to connect the band diagram and the reported electronic transport properties such as Poole-Frenkel conduction [26, 27, 35] and bandtail hopping [36, 37], with suitably careful analysis to help improve our understanding of these materials, expediting the process of it being better evaluated as a candidate material for electronic devices.

\section{EXPERIMENTAL DETAILS AND RESULTS}

\subsection{Film deposition}

a-C films were deposited using pulsed laser deposition (PLD) on n-type $\mathrm{Si}$ substrates (phosphorus doped, $\mathrm{Si}\langle 100\rangle \rho=0.01 \Omega \mathrm{cm}$ ) after using a standard solvent cleaning process, and then subsequently placing the samples inside a vacuum chamber with a base pressure of $\sim 10^{-6}$ Torr. A KrF Lambda Physik Excimer laser (LPX 210i), operating at $248 \mathrm{~nm}$ with $25 \mathrm{~ns}$ full-width half-maximum (FWHM) pulse duration was used as the pulsed UV laser source. The pyrolytic graphite target (Kurt. J. Lesker, $99.99 \%$ pure) was rotated during deposition to avoid ablation of the same spot, and its surface was cleaned using laser ablation prior to deposition using a focused pulse at a fluence of $4 \mathrm{~J} / \mathrm{cm}^{2}$. The repetition rate of the laser pulse was fixed at $10 \mathrm{~Hz}$ for all depositions. The laser fluence was fixed at $4 \mathrm{~J} / \mathrm{cm}^{2}$ for both a-C and a- $\mathrm{CN}_{\mathrm{x}}$ measurements. $\mathrm{N}_{2}$ gas with a pressure of 40 mTorr was introduced to the chamber for a- $\mathrm{CN}_{\mathrm{x}}$ deposition. Both types of a-C and a-CN $\mathrm{C}_{\mathrm{x}}$ films with a thickness of $30 \mathrm{~nm}$ were deposited on the highly doped c-Si substrates. An additional a- $\mathrm{CN}_{\mathrm{x}}$ sample was deposited with the laser fluence of 12 $\mathrm{J} / \mathrm{cm}^{2}$ under a 20 mTorr of $\mathrm{N}_{2}$ gas for UPS measurements of the valence band. The 
sample homogeneity was checked by repeating measurements at different points on the sample.

\subsection{Electron energy loss spectroscopy (EELS)}

A CM200 Philips Supertwin TEM operating at $200 \mathrm{kV}$ with a $\mathrm{LaB}_{6}$ filament was used for measurements with a Gatan Imaging Filter GIF2000 spectrometer for EELS. Films with a thickness of approximately 30nm were floated off from the mica substrate by immersing it in deionized (DI) water, and then the film was placed on copper TEM grids.

Figure 2 shows the EELS carbon $\mathrm{K}$ edges which correspond to the empty conduction band and the curve consists of $\sigma^{*}$ and $\pi^{*}$ bands. The line and dashed curves are for the guide of the eye for $\mathrm{a}-\mathrm{C}$ and $\mathrm{a}-\mathrm{CN}_{\mathrm{x}}$, respectively. Those transitions appears as two peaks; $\pi^{*}$ band $(\sim 284.6 \mathrm{eV})$ and $\sigma^{*}$ band $(\sim 296 \mathrm{eV})$, respectively. The two curves are normalized by the height of the $\sigma^{*}$ band, and the intensity and width of the $\pi^{*}$ band increases by the nitrogen incorporation.

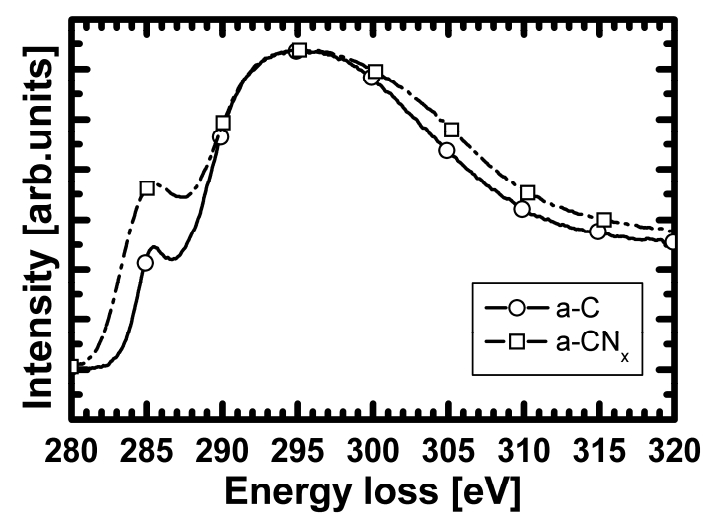

Figure 2: EELS Carbon $\mathrm{K}$ edge which corresponds to the conduction band of a-C and a-CNx films. 
The density, $\mathrm{sp}^{2}$ fraction and the nitrogen content can also be evaluated from the EELS low loss spectra and carbon $\mathrm{K}$ edge, and the details can be seen elsewhere.[30] The $\mathrm{sp}^{2}$ fraction of a-C and a- $\mathrm{CN}_{\mathrm{x}}$ films were found to be $30 \%$ and $60 \%$, respectively. The density of a-C and a- $\mathrm{CN}_{\mathrm{x}}$ films were 2.4 and $1.8 \mathrm{~g} / \mathrm{cm}^{3}$. The nitrogen content of a-CN $\mathrm{CN}_{\mathrm{x}}$ film was 17 at. $\%$.

\subsection{Scanning tunneling spectroscopy (STS)}

STM and STS experiments were performed at room temperature in a commercial UHV-STM (Omicron VT Multiscan STM) equipped with a SEM column using an electrochemically-etched tungsten tip. During the experiment, the base pressure in the UHV chamber was $2 \times 10^{-11}$ mbar. STM images were recorded in the constant current mode, with the sample biased at $+2.5 \mathrm{~V}$ and the tunneling current maintained at approximately $100 \mathrm{pA}$. The STM images were processed and analysed using the WSxM package.[38] The STS I-V curves were taken in the range -5 to $5 \mathrm{~V}$ for at least 16 positions from a $100 \mathrm{~nm} \times 100 \mathrm{~nm}$ image, and are averaged in order to get the DOS corresponding to a large area. Thus, the observation area is large enough to treat the STS results as the bulk nature rather than the local properties.

Figure 3 (a), (b) and (c) show the averaged tunneling current versus the sample bias, $\mathrm{dI} / \mathrm{dV}$ plot and the normalised conductance for a-C and $\mathrm{a}-\mathrm{CN}_{\mathrm{x}}$ films, respectively. The normalised conductance is proportional to the density of state, and a negative sample bias shows the valence band of the films, whereas a positive sample bias shows the conduction band of the films.

It is remarkable that a-C shows a strong peak at approximately $-1.5 \mathrm{~V}$ in the bandstructure, a feature never measured experimentally previously and always 
inferred. More recently such a feature has been attributed to the p-type behaviour found in graphene. The slope of the valence band is steeper than that of conduction band, and it was attributed to the fact that a-C is a slightly p-type semiconductor due to the presence of defects. $[15,16]$ Also a narrowing of the bandgap or an increase of the bandtail can be observed for a- $\mathrm{CN}_{\mathrm{x}}$ films, compared with the a-C films (see figure 3.) The slope at the bottom of the conduction band in the a- $\mathrm{CN}_{\mathrm{x}}$ is steeper than a-C, which may be indicative that the nitrogen atoms acting as shallow donors in the $\pi^{*}$ band of the a-C[14-16]. The bandgap was estimated using the conductance of STS following the method of Arena et al. and the magnified dI/dV curve is shown in Figure 8, and will be discussed later.

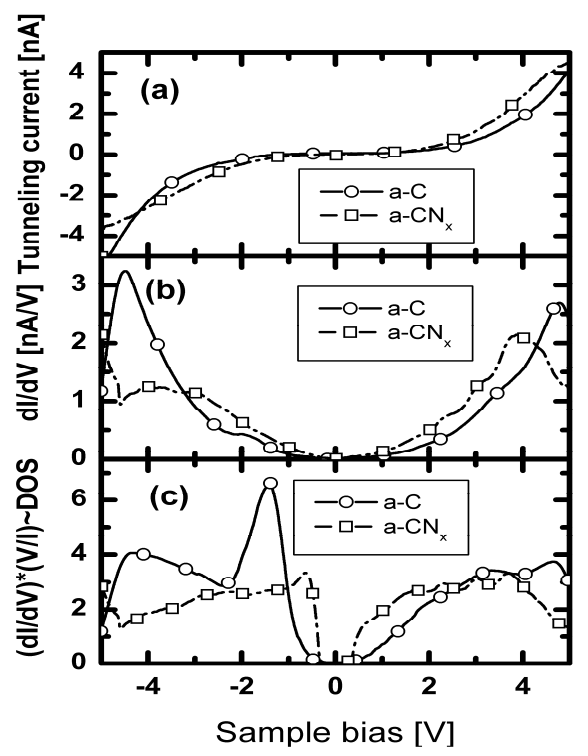

Figure 3: STS spectra of a-C and a-CNx films; (a) Tunnelling current, (b) conductance and (c) normalised conductance which is proportional to DOS versus sample bias. 


\subsection{Photoelectron spectroscopy (XPS and UPS)}

An Omicron Multiprobe UHV system equipped with an Omicron EA125 analyzer was used for both X-ray photoelectron spectroscopy (XPS) and ultraviolet photoelectron spectroscopy (UPS) experiments. For XPS, spectra were acquired with a pass energy of $50 \mathrm{eV}$ and $20 \mathrm{eV}$ for survey and higher resolution respectively, using $\mathrm{Mg} \mathrm{K} \alpha$ radiation $(\mathrm{hv}=1256.4 \mathrm{eV}$ ) from a VG XR3E2 twin anode source. The base pressure was in the range of $10^{-10}$ mbar for the entire set of experiments.

For UPS, He I (21.22 eV) from an Omicron HIS13 windowless He lamp was used. The pass energy was set to $5 \mathrm{eV}$ for a $2 \mathrm{~mm}$ diameter analysis area defined by the entrance slit to the analyzer. Spectra were normalized to the maximum intensity of the inelastically scattered electrons. The work function was evaluated from the cut-off of the secondary electrons with the background. The samples were biased at $-3 \mathrm{~V}$ for the work function measurements in order to accelerate the low kinetic energy electrons. The work function of a-C and a- $\mathrm{CN}_{\mathrm{x}}$ films so determined were $5.0 \pm 0.1 \mathrm{eV}$ and $5.1 \pm 0.1 \mathrm{eV}$, respectively. These values are consistent with previous calculations of work functions and the derivation of their band structures that describe the a-C:n-Si heterojunction.[39]

Samples were introduced to the UHV chamber immediately after deposition. XPS/UPS measurements were carried out without Ar+ sputtering to avoid inducing changes to the samples due to the graphitization and nitrogen loss[12]. Although the UPS spectra close to the Fermi level might be affected by oxygen contamination,[12] these spectra should still provide relevant information about the valence band states and its influence on conduction.[11] Any oxidation would be 
minimal and conduction perpendicular to such a mono-layer would have a minimal influence on the measured data under the electric fields used.

Figure 4 presents the UPS valence band spectra of both a-C and a- $\mathrm{CN}_{\mathrm{x}}$, and they both show peaks associated with the $\pi$ and $\sigma$ valence bands. The peaks around $14 \mathrm{eV}$ in Figure 4 (a) and $16 \mathrm{eV}$ in Figure 4 (b) are attributed to the inelastically scattered, secondary electrons. However, no peaks corresponding to the carbonnitrogen bonds were found in these spectra, possibly due to the relatively low nitrogen content, estimated at $17 \%$ by EELS measurements.

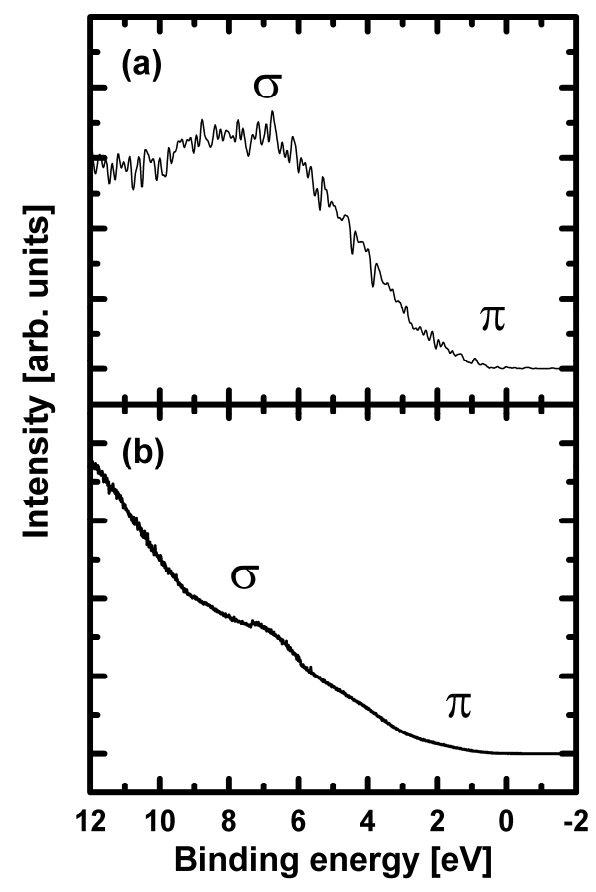

Figure 4: UPS valence spectra (a) a-C and (b) a-CNx. 
The films were exposed to air before loading to the UPS chamber, and therefore oxygen and water contamination of the surface is expected. Thus, the $\mathrm{Ar}^{+}$ ion etching was carried out for a-C and a- $\mathrm{CN}_{\mathrm{x}}$ films. In this case, higher nitrogen content $(25 \%)$ a- $\mathrm{CN}_{\mathrm{x}}$ films were deposited with higher laser fluence $\left(12 \mathrm{~J} / \mathrm{cm}^{2}\right)$ in order to confirm the position of the $\mathrm{C}-\mathrm{N}$ related peaks on valence band. Figure 5 (a) and (b) show the UPS signals of a-C without and with $\mathrm{Ar}^{+}$ion etching, respectively. Figure 5 (c) and (d) are the UPS spectra of a- $\mathrm{CN}_{\mathrm{x}}$ without and with $\mathrm{Ar}^{+}$ion etching, respectively. The UPS measurements show new peaks $(\sim 5 \mathrm{eV}$ and $\sim 7 \mathrm{eV})$ remaining even after the $\mathrm{Ar}^{+}$sputtering, which may correspond to the bonds between carbon and nitrogen atoms, on these higher nitrogen content films as shown in Figure 5 (d), and discussed later. 

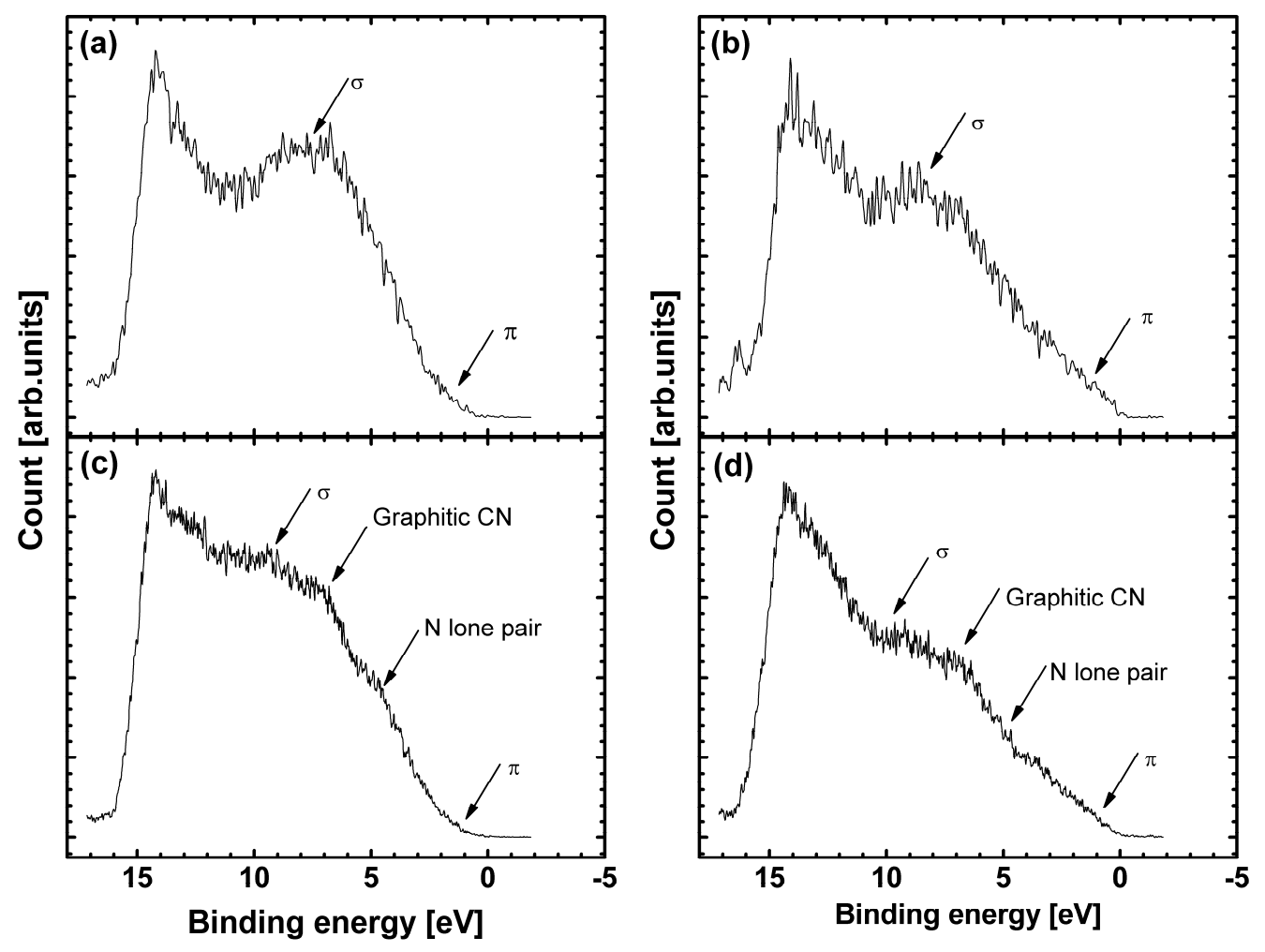

Figure 5: UPS valence spectra (a) a-C, (b) a-C after Ar sputtering, (c) a-CNx with the laser energy $12 \mathrm{~J} / \mathrm{cm} 2$ and 20 mTorr of Nitrogen and, (d) a-CNx after Ar+ sputtering. The sputtering was performed at $5 \mathrm{keV}$, for 1 minute.

\subsection{UV-VIS-NIR optical transmittance}

The Tauc optical bandgap $\left(\mathrm{E}_{\mathrm{g}}\right)$ and the energy where the absorption coefficient is $10^{4} \mathrm{~cm}^{-1}\left(\mathrm{E}_{04}\right)$, extracted from UV-VIS-NIR measurements, have been used to evaluate the optical bandgap of amorphous semiconductors.[2] The Tauc gap is obtained by plotting $\sqrt{\alpha E}$ which assumes the parabolic DOS close to the edge, where $\alpha$ is the absorption coefficient and $E$ is the photon energy, as a function of photon energy $\mathrm{E}$. [40] The $\mathrm{E}_{04}$ is evaluated from the absorption versus photon energy plot. These two optical gap values are correlated in a-C [33] and 
related to be the transition between $\pi-\pi^{*}$ bands (JDOS). However, sometimes $\mathrm{E}_{04}$ cannot be obtained using UV-VIS when the optical gap is narrow, whereas $\mathrm{E}_{\mathrm{g}}$ can be obtained from UV-VIS.[41] Thus, $\mathrm{E}_{\mathrm{g}}$ was evaluated for the comparison in this study.

A Cary 5000, Varian ultraviolet-visible-near infrared (UV-VIS-NIR) spectrometer was used in the dual beam mode for the optical transmittance measurements in the range between $190 \mathrm{~nm}(\sim 6.5 \mathrm{eV})$ and $3000 \mathrm{~nm}(\sim 0.4 \mathrm{eV})$. 30nm thick films deposited on quartz substrates were used for these measurements.

The transmittance spectra were converted to the absorption coefficient versus photon energy, and then converted to Tauc plot data. From the Tauc plots as shown in Figure 6, $\mathrm{E}_{\mathrm{g}}$ were obtained, and shown to be $0.5 \mathrm{eV}$. Normally, the region of $(\alpha E)^{1 / 2}$ with values between $200-400\left[\mathrm{~cm}^{-1} \mathrm{eV}\right]^{1 / 2}$ is used for the linear fit as shown in Figure 6(a). The intercept of the fitted line and the $\mathrm{x}$ axis is used as the Tauc gap. The whole image of the Tauc plot is also shown as Figure 6 (b).

The optical bandgaps are smaller than $1 \mathrm{eV}$ for both a-C and a- $\mathrm{CN}_{\mathrm{x}}$ films. This bandgap value is quite small, especially for the a-C films with $70 \% \mathrm{sp}^{3}$ fraction. However, non-hydrogenated a-C deposited by sputtering can show small optical band gaps (less than $1 \mathrm{eV}$ ) with a high $\mathrm{sp}^{3}$ fraction as reported due to the stress induced $\mathrm{sp}^{2}$ to $\mathrm{sp}^{3}$ transitions[42]. However, the $\mathrm{sp}^{3}$ fraction is still high, and stress induced localized states reduce the energy of the Tauc optical gap to less than $1 \mathrm{eV}$. The Tauc optical bandgap increases due to nitrogen incorporation, reducing the internal stress in this case.[27, 35] 

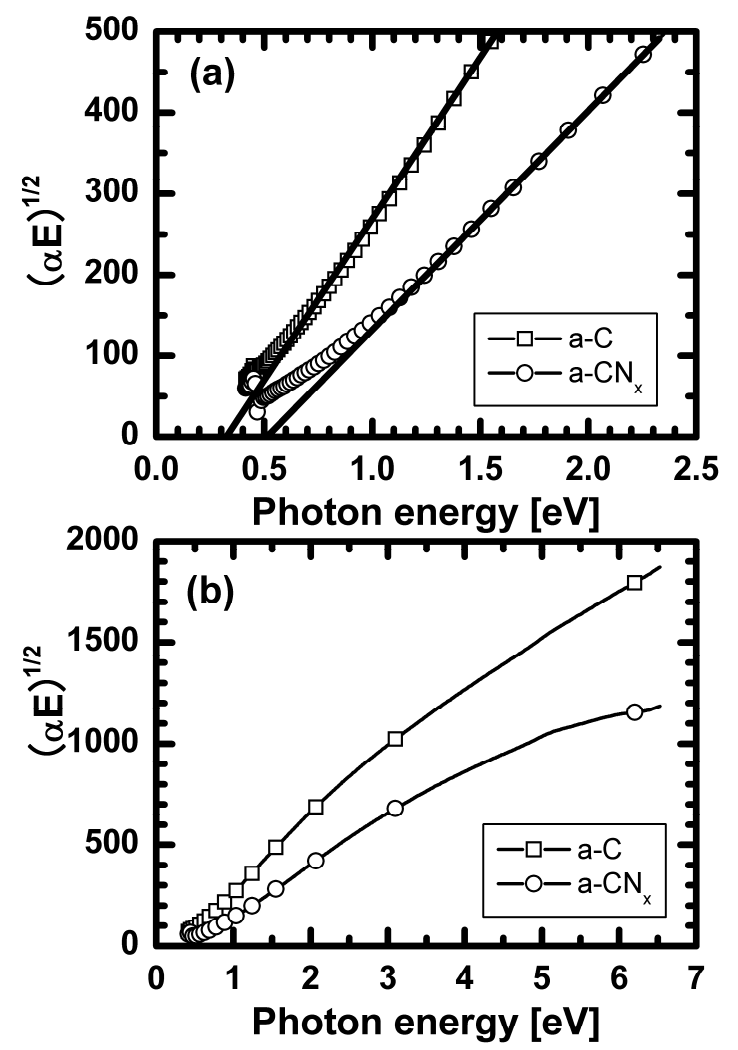

Figure 6: Tauc plot (a) The region where Tauc gap is determined and (b) the whole plot of the photon energy range between $0.5-6.5 \mathrm{eV}$.

\subsection{Photothermal deflection spectroscopy (PDS)}

PDS is the measurement of the absorption coefficient using the "mirage effect' $[43,44]$. Under light exposure, electrons are promoted from the valence to the conduction bands, at which point this energy is converted into thermal energy at room temperature. A thermal gradient is created at the exposed spot and consequently the refractive index of the gas or the liquid in the vicinity of the surface of the sample is modulated. This effect can be detected as a deflection of a laser beam probe passing next to the surface, within the thermal diffusion length. 
A halogen lamp was used as a light source, and the incident light was focused onto the sample surface through a monochromatic meter (CIV LASER CM110) and a mechanical chopper. The samples were dipped in the tetrachloroethane $\left(\mathrm{CCl}_{4}\right)$ and the refractive index fluctuations in the vicinity of the exposed spot were detected using a semiconductor laser (LT015MF, Sharp) with a wavelength of $830 \mathrm{~nm}$. The deflection of the probe laser was monitored by a photo detection device (S3931, Hamamatsu photonics) equipped with a lock-in-amplifier (SR830 by Stanford research system).

The PDS signal was fitted with the absorption coefficient versus photon energy plot evaluated from UV-VIS-NIR optical transmittance as shown in Figure 7. The PDS data is shown every 15 points for clarity. PDS and optical transmittance spectra are consistent and exhibit a bandgap smaller than $2 \mathrm{eV}$, which indicates that the narrow optical bandgap $(<1 \mathrm{eV})$ is not related to the optical measurement setup. It also indicates that the surface condition is similar to the bulk properties since PDS is a relatively surface sensitive technique and UV-VIS-NIR optical transmittance is not, and therefore the oxygen contamination does not significantly affect the insides the film due to its relatively high density. The effect of oxygen or water at the surface layer could be removed by $\mathrm{Ar}^{+}$ion etching for the UPS measurements, and these results coincide with the results of optical measurements. 


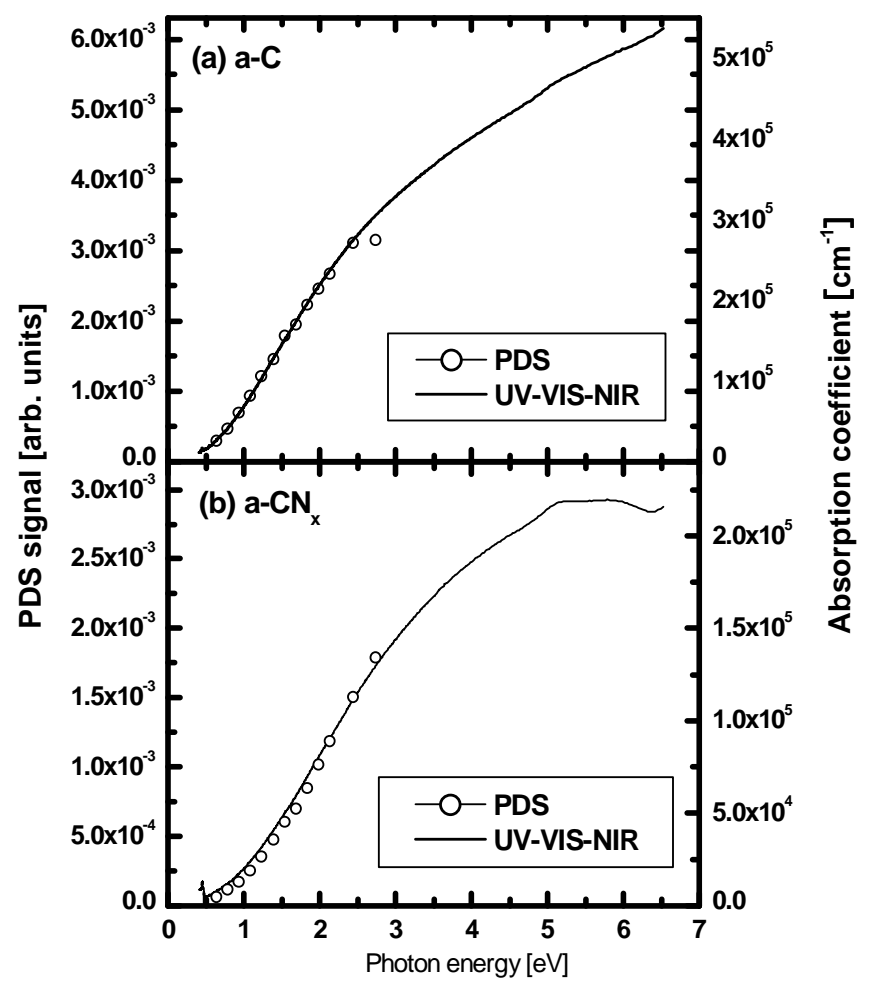

Figure 7: The photon energy dependence of the PDS signal and the absorption coefficient obtained from UV-VIS-NIR optical transmittance (a) a-C and (b a-CNx). The line is the UV-VIS-NIR spectra and the dots are the PDS signal. The PDS signal is shown for every 15 points for clarity.

This result is contrary to the high $\mathrm{sp}^{3}$ fraction film with high optical bandgaps. Normally, the high $\mathrm{sp}^{3}$ fraction film has a smaller contribution from the $\pi$ and $\pi^{*}$ bands, and thus the optical bandgap of the high $\mathrm{sp}^{3}$ fraction films deposited by the filtered vacuum cathodic arc (FVCA) can reach up to $3.6 \mathrm{eV}\left(\mathrm{E}_{04}\right)$.[41] However, the distribution of the $\mathrm{sp}^{2}$ states, their possible clustering and the 
percolation of the states need to be taken into consideration when discussing this result.

\section{Discussion}

The effect of the nitrogen atom incorporation in the band structure of the host amorphous carbon will be visible to all measurements performed; EELS, STS and UPS, which then should provide information on the shape of the conduction band, close to the Fermi level, and valence band. The change on the bandstructure due to the presence of bonding between carbon and nitrogen, and therefore the change of JDOS corresponding to the transitions of electrons from the filled valence to the empty conduction bands, were also detected by optical transmittance and photothermal deflection spectroscopy.

The shapes of the EELS carbon $\mathrm{K}$ edges of a-C and $\mathrm{a}-\mathrm{CN}_{\mathrm{x}}$ films are different, corresponding to modifications of the conduction band induced by nitrogen incorporation. a- $\mathrm{CN}_{\mathrm{x}}$ films with a nitrogen content of 17 at. \% shows a larger $\pi^{*}$ peak and a corresponding increase in the $\mathrm{sp}^{2}$ fraction that is expected as the nitrogen atoms tend to bond preferentially with $\mathrm{sp}^{2}$ hybridized carbon atoms.[22, 45] Waidmann et al. exhibited the EELS analysis of a-C and a- $\mathrm{CN}_{\mathrm{x}}$ having similar

$\mathrm{sp}^{2}$ deposited by FVCA, and the broadening of $\pi^{*}$ peak, reduction of optical bandgap and increase of the $\mathrm{sp}^{2}$ fraction was also observed due to the nitrogen incorporation. [46]

Nitrogen incorporation increases the width of the $\pi^{*}$ band, which is consistent with the decrease of the bandgap observed by STS measurements (see 
Figure $3 \mathrm{c}$ ). The increase of the width of the $\pi^{*}$ peak is also attributed to the C-N bondings.[22] The DOS in the vicinity of the Fermi level can be obtained by plotting the normalised conductance resulting from STS measurements. The nitrogen incorporation increases the slope of the conduction band compared with the a-C, and the electrical gap of a-CN $\mathrm{x}_{\mathrm{x}}$ appears to be smaller than that of a-C.

However, the Tauc gap is slightly increased $(\sim 0.2 \mathrm{eV})$ by nitrogen incorporation, which could imply a mopping up of defect tail states from the material. This is consistent with the previously observed reduction in the electron spin resonance signal with nitrogen incorporation, that is accompanied by a reduction in the compressive stress in the films.[47] Moreover, the sharp peak in the case of a-C films may be attributed to the stress induced localised states[35], which are not observed for $\mathrm{a}-\mathrm{CN}_{\mathrm{x}}$.[27] Nitrogen incorporation giving rise to an under constrained amorphous network which may have arisen partly due to the reduction of the impact velocity of carbon growth species due to scattering from $\mathrm{N}_{2}$ molecules at elevated growth pressures,[35] probably decreases the stress in the film. (see Figure 3(c)) A shorter bandtail observed in a- $\mathrm{CN}_{\mathrm{x}}$ films, and the sharp peak in the DOS at approximately $1.5 \mathrm{eV}$ for a-C could be attributed to the stress induced localized states as shown in Figure 3(c)[29].

The optical bandgap is normally narrower than the electrical mobility gap. Arena et al. determined the bandgap using dI/dV plot of STS, which is defined as the intercepts of $\mathrm{dI} / \mathrm{dV}$ at the valence and conduction band slopes in the region $\pm 2 \mathrm{eV}$ away from the Fermi level[15, 16]. Based on this definition, the band gaps of PLD a-C and a- $\mathrm{CN}_{\mathrm{x}}$ are estimated at approximately $2 \mathrm{eV}$ from STS measurements as shown in Figure 8, and is consistent with previous reports.[1, 2] However, the 
optical bandgap is much smaller than the bandgap measured by STS, of which difference can be attributed to the long bandtail.[35] If the bandgap obtained by STS rather close to the bandgap attributed to the transition between the edge of $\sigma$ and $\sigma^{*}$ bands or $\pi$ and $\pi^{*}$ bands without long bandtail, the electrical bandgap between the mobility edge of valence and conduction bands are larger then $2 \mathrm{eV}$. This is consistent with the simulations in which it is shown that the $\pi$ and $\pi^{*}$ states are highly localised.[8] Then, the strong peak around $-1.5 \mathrm{eV}$ as shown in Figure 3 (c) could be associated with the defects band, which was expected by the electrical conduction results with the Pool-Frenkel model.[29]

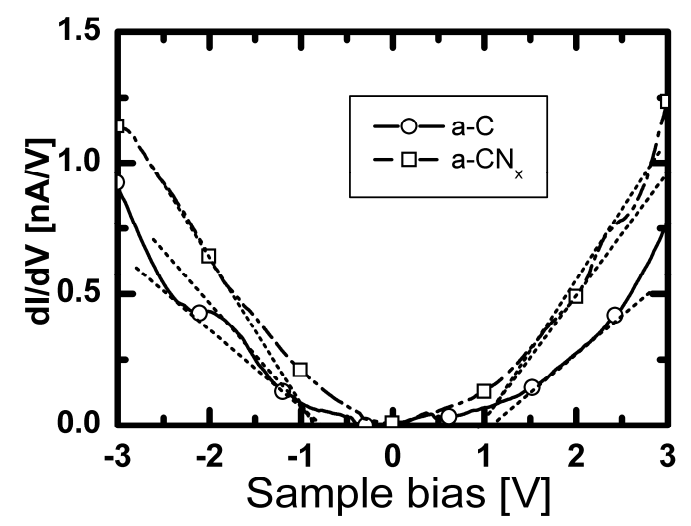

Figure 8: $\mathrm{dI} / \mathrm{dV}$ plots for estimating the bandgap in the a-C and a-CNx.

It was found that films with higher nitrogen content $(25 \%$ as determined by EELS measurements), deposited at higher laser fluences $\left(12 \mathrm{~J} / \mathrm{cm}^{2}\right)$, exhibit additional contributions in the UPS spectra compared with a-C, as shown in Figure 5. These contributions could be attributed to the nitrogenated graphitic clusters $(\sim 7$ 
$\mathrm{eV}$ and $\sim 10 \mathrm{eV})$ and nitrogen lone pair states $(\sim 5 \mathrm{eV})$, in good agreement with the work of Souto et al. who reported UPS measurements and simulation on nonhydrogenated a-CN $\mathrm{CN}_{\mathrm{x}}$ films (nitrogenated graphite clusters and $\beta-\mathrm{C}_{3} \mathrm{~N}_{4}$ ).[17] Peaks at $\sim 8 \mathrm{eV}$ and at less than $4 \mathrm{eV}$ could be attributed to carbon $\sigma$ and $\pi$ bands, respectively. These peak assignments are consistent with the chemical bonding configurations formed by carbon and nitrogen atoms.[22]

Upon $\mathrm{Ar}^{+}$sputtering, small peaks corresponding to carbon-nitrogen bonds (C-N peaks) appeared. XPS measurements show that the nitrogen content decreases after $\mathrm{Ar}^{+}$sputtering. It is thought that the nitrogen was selectively etched, as shown in similar studies.[12] Since the mass of nitrogen and carbon atoms are comparable, the physical etching rate should be similar. However, the nitrogen loss implies that the nitrogen preferentially etches. It does not necessarily mean that the bonds between carbon and nitrogen atoms are disassociated. There is a possibility of a break at the bonds between $\mathrm{C}$ and $\mathrm{CN}$, and in such a case, nitrogen loss can still occur.

Compared to a-C, the UPS valence band spectra of a- $\mathrm{CN}_{\mathrm{x}}$ films do not show significant changes for films containing 17 at.\% of nitrogen (laser fluence of 4 $\mathrm{J} / \mathrm{cm}^{2}$ ), whereas the a- $\mathrm{CN}_{\mathrm{x}}$ films containing 25 at.\% of nitrogen show peaks which can originate from $\mathrm{CN}$ bonding. The intensity of the carbon $\sigma$ band of a- $\mathrm{CN}_{\mathrm{x}}$ appears to be weaker than for a-C, and seems to correspond to a reduction in the quantity of $\mathrm{sp}^{3}$ hybridized carbon. The higher fluence laser can create larger numbers of highly energetic carbon atoms and ions, enough to react with $\mathrm{N}_{2}$ in the vacuum chamber. Consequently, higher laser fluences form higher nitrogen content 
films, and thus higher nitrogen content films show stronger peaks which correspond to $\mathrm{CN}$ and nitrogen lone pair peaks.

Furthermore, even if the peaks associated with carbon and nitrogen bonding are underestimated on the $\mathrm{Ar}^{+}$sputtered samples due to the nitrogen loss and the graphitization, it is possible to detect a DOS modulation due to the nitrogen incorporation on the band structure of a- $-\mathrm{CN}_{\mathrm{x}}$ as shown in Fig. 4 and 5. Of course, a higher degree of DOS modulation is expected in the samples without $\mathrm{Ar}^{+}$sputtering. In this study, the position of the $\mathrm{CN}$ related peaks are important in addition to the actual DOS shape. Thus, even though the samples are exposed to air for a few minutes, the result of the UPS measurement is still important and valid. Futhremore, the PDS and optical transmittance results coincided, which implies that the surface oxygen contamination is not extremely high, and therefore the UPS results are still reliable.

Considering the fact that this material has an electrical mobility band gap which corresponds more closely with the $\sigma$ and $\sigma^{*}$ bands, the Poole-Frenkel conduction reported for electrical conduction at high electric fields[27, 29, 35] may occur at the edge of the extended states ( $\sigma$ or $\sigma^{*}$ band). Then, the neutral trapping centres which provide electrons to the extended states under a high electric field should exist within a defect band or localized states ( $\pi$ or $\pi^{*}$ band). $\mathrm{Sp}^{2}$ clusters appear to be a possible origin for these neutral trapping centres.[28] The neutral trapping centres which are located around 0.2-0.4 eV far from the edge of the extended state ( $\sigma$ or $\sigma^{*}$ band) according to the activation energy studies, will correspondingly be in the delocalised states ( $\pi$ or $\pi^{*}$ band) close to the edge of the extended states ( $\sigma$ or $\sigma^{*}$ band). This is consistent with the published simulation 
studies[8] since the $\pi$ and $\pi^{*}$ bands are localised and will thus allow for pinned energy levels and states.

The presence of a long tail is also in agreement with a conduction mechanism at low fields involving bandtail hopping. Here, the $\mathrm{sp}^{2}$ clusters are thought to act as hopping centres.[28] The expected band structure and the positions of states corresponding to the two conduction mechanisms, bandtail hopping at low fields and Poole-Frenkel conduction at high fields are entirely consistent with the DOS we currently propose for the films, and further corroborates our analysis. If the electrical bandgap is at least more than $2 \mathrm{eV}$, as evaluated by STS, the conduction band can be attributed to the $\sigma^{*}$ band.

With significant nitrogen incorporation, the $\pi$ and $\pi^{*}$ states are still localized, and any delocalized carriers in the $\sigma$ and $\sigma^{*}$ states correspond to the valence and the conduction bands, respectively. Thus, the reported conduction mechanisms in a$\mathrm{CN}_{\mathrm{x}}$ films, the bandtail hopping and Poole-Frenkel (PF) conduction, are considered to occur in a similar manner to that reported for a-C.

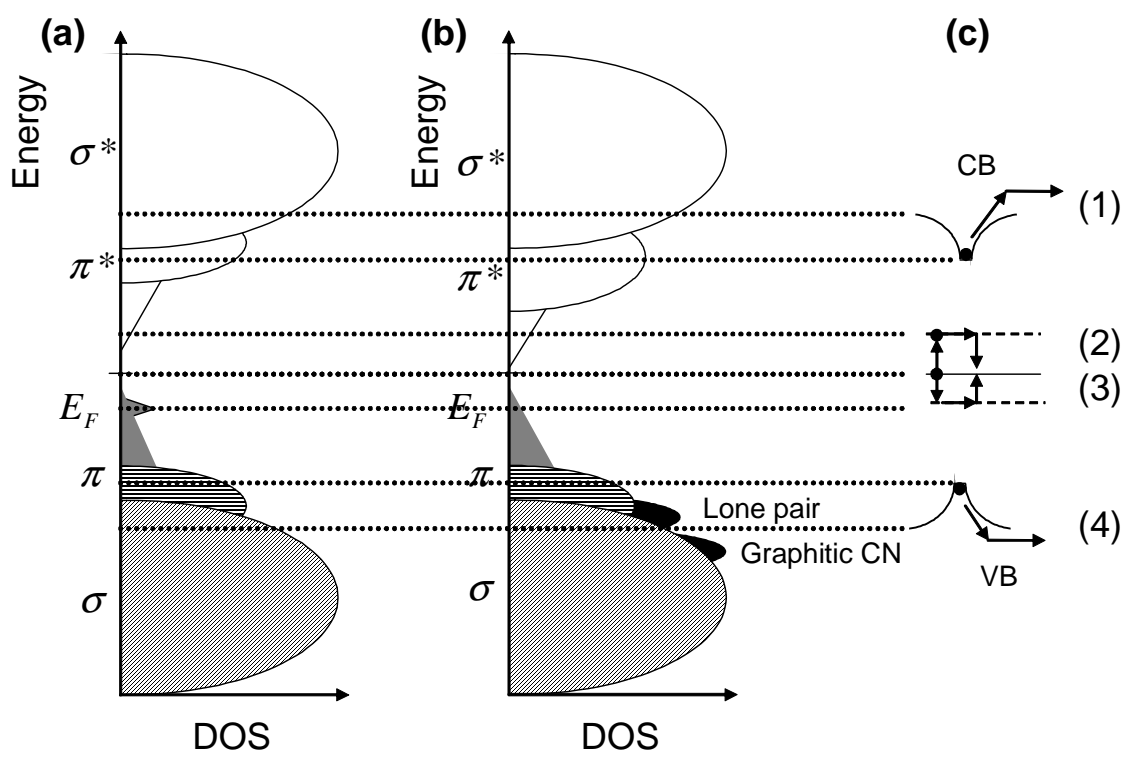


Figure 9: Schematic diagram on the model of the band structure for PLD (a) a-C and (b) a-CNx film. (c) Conduction mechanism at low and high fields.

However, the PF model does not provide evidence for the carrier type in the material. The possible main conduction mechanisms that can give rise to charge movement in amorphous materials are four types; (1) PF hopping through the bottom of the extended states ( $\sigma^{*}$ band) in the conduction band, (2) band tail hopping above the Fermi level, (3) band tail hopping below the Fermi level, and, (4) PF hopping through the top of the extended states ( $\sigma$ band) in the valence band. The band diagram and the possible conduction mechanisms for a-C and a- $\mathrm{CN}_{\mathrm{x}}$ are illustrated in Figure 9.

The conduction mechanism of both a-C and a- $\mathrm{CN}_{\mathrm{x}}$ films appear to be PooleFrenkel type at high electric fields $[27,35]$. Thus, the conduction is believed to be through the edge of the extended states, and the contact is negligible since it is bulk limited. Using the three terminal device structures, the gate voltage can modulate the conduction between the source and the drain [26]. For both a-C and a-CN $\mathrm{CN}_{\mathrm{x}}, \mathrm{p}-$ channel depletion like behaviours were observed, and therefore the carrier type appears to be holes for both a-C and a- $\mathrm{CN}_{\mathrm{x}}$.

The result for the a-C device mentioned above is consistent with previous reports of three terminal devices at low electric fields [23, 24]. In the previous reports, it was explained that a-C has a defect band below the Fermi level, with bandtail hopping occuring through these defects, and the Fermi level is modulated by the applied gate voltage. 
The above result of the a- $\mathrm{CN}_{\mathrm{x}}$ device contradicts former reports which pointed out that a- $\mathrm{CN}_{\mathrm{x}}$ is an n-type channel semiconductor[48] and nitrogen works as a shallow donor with respect to the defect band in the $\pi^{*}$ states, but is still deep with respect to $\sigma^{*}$ band. $\mathrm{N}$-doping may improve the mobility of a- $\mathrm{CN}_{\mathrm{x}}$ when the transport mechanism is within the bandtail hopping phase, and not at the extended states edge.

However, the carrier type was p-type for both the a-C and a- $\mathrm{CN}_{\mathrm{x}}$ films used this study. Therefore, the possible conduction models (4) illustrated in Figure 9 are the conduction mechanisms proposed for $\mathrm{a}-\mathrm{C}$ and $\mathrm{a}-\mathrm{CN}_{\mathrm{x}}$ films at high electric fields. If the strong peak observed in the STS measurement is the defects band, it can provide electrons to the edges of the extended states ( $\sigma$ band) as the neutral trapping centres.

The STS result also coincides with the results of TFT measurements. Then the mobility edge of a-C is thought to be around $-2 \mathrm{eV}$, and therefore the electrical bandgap between the mobility edges of valence and conduction bands is probably around $4 \mathrm{eV}$. Due to the nitrogen incorporation, this strong peak disappears but the depth of the neutral trapping centre is almost the same $(\sim 0.18 \mathrm{eV}$ for a-C deposited by $4 \mathrm{~J} / \mathrm{cm}^{2}$ and $0.16 \mathrm{eV}$ for $\left.\mathrm{a}-\mathrm{CN}_{\mathrm{x}}\right)[27,29]$. This is probably because the new nitrogen origin peak such as the $\mathrm{N}$ lone pair and the Graphitic $\mathrm{CN}$ appear much closer to the Fermi level rather than the $\sigma$ peak of a-C film as observed by UPS, in addition to the narrowing of the gap between the band tails detected by STS measurements as shown in Figure 3 (c).

It has been reported that nitrogen may act as a weak donor in the compensation mode within the $a-\mathrm{CN}_{\mathrm{x}}$ structure and is a weakly n-type 
semiconductor. These new results do not support this hypothesis, and the UPS signal does not show significant peaks when the nitrogen pressure is 20 mTorr, whereas there are two new peaks with higher nitrogen content films. STS data also indicate that the Fermi level is still in midgap for both a-C and a-CN $\mathrm{N}_{\mathrm{x}}$. Llie et al. [49] showed using the Kelvin prove that the surface Fermi level is pinned and insensitive for any N-doping. Thus, there is also a possibility that the film is still ptype due to the low nitrogen content in the films.

The effective mobilities were also estimated using three terminal devices; a$\mathrm{C}$ is $1.1 \times 10^{-3} \mathrm{~cm}^{2} \mathrm{~V}^{-1} \mathrm{~s}^{-1}$ and a- $\mathrm{CN}_{\mathrm{x}}$ is $9.6 \times 10^{-4} \mathrm{~cm}^{2} \mathrm{~V}^{-1} \mathrm{~s}^{-1}$ [26]. These values are more than one order higher than those previously reported for a-C/a-CN $\mathrm{C}_{\mathrm{x}}$ TFTs. This is because the conduction is via the edge of the extended states in the valence band by holes. Therefore, the effective mobility can be much higher than the effective mobility of hopping.

This result implies that there is a possibility to improve the effective mobility to more than $10^{-3} \mathrm{~cm}^{2} \mathrm{~V}^{-1} \mathrm{~s}^{-1}$ if the carriers are provided into the extended states of carbon films. Furthermore, reducing the localized states including the long bandtail within the gap between the $\sigma-\sigma^{*}$ bands should improve the effective mobility at the extended states, resulting in a longer mean free path.

One possible approach is a- $\mathrm{CN}_{\mathrm{x}}$ films with higher nitrogen content (N/C 1).[25] The optical bandgap of such a-CN $\mathrm{CN}_{\mathrm{x}}$ films is almost $3 \mathrm{eV}$, which implies that reduction of the $\pi$ and $\pi^{*}$ bands, and possible new peaks originate from the nitrogen lone pair and the Graphitic C-N bonds. In such band structured films, a higher effective mobility is expected. 
This analysis including the prediction of a possible effective mobility of more than $10^{-3} \mathrm{~cm}^{2} \mathrm{~V}^{-1} \mathrm{~s}^{-1}$ using carbon based films can be achieved by the careful consideration of the whole DOS measurements using several techniques on one sample. Based on this analysis, we believe that the carbon based films could be a next generation electronic material.

\section{Conclusion}

The band structure of PLD a-C and the effect of nitrogen incorporation were studied using STS, UPS and EELS. The results are consistent with optical transmittance and PDS measurements. The band structure suggests that a-C is slightly p-type due to the presence of defects. A strong peak at around $-1.5 \mathrm{eV}$ was observed on a-C film by STS measurements.

The effect of nitrogen on the valence band was detected in the UPS spectra. For high nitrogen contents (25 at.\%), peaks corresponding to $\mathrm{C}-\mathrm{N}$ bonding and to the $\mathrm{N}$ lone pair were observed. According to EELS measurements, the conduction band is less affected, with increases only in the $\mathrm{sp}^{2}$ fraction observed.

A transport model for PLD a-C and a-CN $\mathrm{N}_{\mathrm{x}}$ films which satisfies the reported electrical conduction mechanism at low and high fields is postulated. The conduction mechanism at high fields appears to correspond to the Poole-Frenkel effect established at the top of the valence band. Neutral trapping centres appear to be located at the bottom of the $\pi$ band and the hopping sites are situated in the long tail in the bandgap. The peak in the DOS below the Fermi level at $-1.5 \mathrm{eV}$ may contribute to the low electric field conduction (band tail hopping), and may contribute as the neutral trapping centres at high electric field if the carrier type is 
holes. It agrees with a former report which showed a p-type channel. The DOS/JDOS analysis implies that a-C/CN ${ }_{x}$ can be considered as a potential next generation electronic material if the hole conduction at the edge of the extended states can be used for electrical conduction.

\section{Acknowledgements}

The authors would like to thank EPSRC for financially supporting this work in the form of a Portfolio Partnership award to the University of Surrey.

\section{References}

[1] Edited by S.R.P. Silva et. al., Amorphous carbon: state of the art. Singapore: World scientific; 1997.

[2] Edited by S.R.P. Silva, Properties of Amorphous carbon. London: INSPEC publication IEE; 2003.

[3] Robertson J. Diamond-like amorphous carbon. Materials Science \& Engineering R-Reports. 2002;37(4-6):129-281.

[4] Bhattacharyya S, Henley SJ, Mendoza E, Gomez-Rojas L, Allam J, Silva SRP. Resonant tunnelling and fast switching in amorphous-carbon quantum-well structures. Nature Materials. 2006;5(1):19-22.

[5] Moseler M, Gumbsch P, Casiraghi C, Ferrari AC, Robertson J. The ultrasmoothness of diamond-like carbon surfaces. Science. 2005;309(5740):1545-8.

[6] Aono M, Nitta S, Katsuno T, Itoh T, Nonomura S. Characterization of low dielectric constant amorphous carbon nitride films. Applied Surface Science. 2000;159:341-4.

[7] Aono M, Nitta S. High resistivity and low dielectric constant amorphous carbon nitride films: application to low-k materials for ULSI. Diam Relat Mater. 2002;11(3-6):1219-22.

[8] Robertson J. Electronic structure of diamond-like carbon. Diam Relat Mater. 1997;6(2-4):212-8.

[9] Robertson J. Electronic and atomic structure of diamond-like carbon. Semicond Sci Tech. 2003;18(3):S12-S9.

[10] Reinke P, Garnier MG, Oelhafen P. In situ photoelectron spectroscopy analysis of tetrahedral amorphous carbon films. Journal of Electron Spectroscopy and Related Phenomena. 2004;136(3):239-45.

[11] Bhattacharyya S, Spaeth C, Richter F. Valence band spectra of nitrogen incorporated amorphous carbon films. J Appl Phys. 2001;89(4):2414-21. 
[12] Kildemo M, Lacerda M, Ballutaud D, Godet C, Raaen S. Experimental study of surface electronic properties of ECR-PECVD deposited a-C1-xNx : H films using UPS, XPS and spectroscopic ellipsometry. Journal of Non-Crystalline Solids. 2002;299:912-6.

[13] Reinke P, Oelhafen P. Electronic properties of diamond/nondiamond carbon heterostructures. Physical Review B. 1999;60(23):15772-81.

[14] Silva SRP, Robertson J, Amaratunga GAJ, Rafferty B, Brown LM, Schwan J, et al. Nitrogen modification of hydrogenated amorphous carbon films. J Appl Phys. 1997;81(6):2626-34.

[15] Arena C, Kleinsorge B, Robertson J, Milne WI, Welland ME. Electronic and topographic structure of ta-C, ta-C : $\mathrm{N}$ and ta-C : B investigated by scanning tunnelling microscopy. Diam Relat Mater. 1999;8(2-5):435-9.

[16] Arena C, Kleinsorge B, Robertson J, Milne WI, Welland ME. Electronic properties of tetrahedral amorphous carbon investigated by scanning tunneling microscopy. J Appl Phys. 1999;85(3):1609-15.

[17] Souto S, Pickholz M, dos Santos MC, Alvarez F. Electronic structure of nitrogen-carbon alloys (a-CNx) determined by photoelectron spectroscopy. Physical Review B. 1998;57(4):2536-40.

[18] Hammer P, Victoria NM, Alvarez F. Electronic structure of hydrogenated carbon nitride films. Journal of Vacuum Science \& Technology a-Vacuum Surfaces and Films. 1998;16(5):2941-9.

[19] Nitta S, Aono M, Katsuno T, Naruse Y. Amorphous carbon nitride deposition by nitrogen radical sputtering $\mathrm{C}+\mathrm{N}+\mathrm{O}+\mathrm{H}$. Diamond and Related Materials. 2003;12(2):219-26.

[20] Fanchini G, Ray SC, Tagliaferro A. Optical properties of disordered carbonbased materials. Surf Coat Tech. 2002;151:233-41.

[21] Alibart F, Drouhin OD, Benlahsen M, Muhl S, Rodil SE, Camps E, et al. Comparison and semiconductor properties of nitrogen doped carbon thin films grown by different techniques. Applied Surface Science. 2008;254(17):5564-8.

[22] Rodil SE, Muhl S. Bonding in amorphous carbon nitride. Diam Relat Mater. 2004;13(4-8):1521-31.

[23] Clough FJ, Milne WI, Kleinsorge B, Robertson J, Amaratunga GAJ, Roy BN. Tetrahedrally bonded amorphous carbon (ta-C) thin film transistors.

Electronics Letters. 1996;32(5):498-9.

[24] Maeng SL, Uchikoga S, Clough FJ, Tagliaferro A, Flewitt AJ, Robertson J, et al. A carbon based bottom gate thin film transistor. Diam Relat Mater. 2000;9(36):805-10.

[25] Habuchi H, Koketsu H, Katsuno T. Optical properties of amorphous carbon nitride films with high nitrogen content. Diam Relat Mater. 2007;16(4-7):1340-2.

[26] Miyajima Y, Shkunov M, Silva SRP. Amorphous carbon and carbon nitride bottom gate thin film transistors

Appl Phys Lett. 2009;95:102102.

[27] Miyajima Y, Adamopoulos G, Henley SJ, Stolojan V, Tison Y, GarciaCaurel E, et al. Electronic state modification in laser deposited amorphous carbon films by the inclusion of nitrogen. J Appl Phys. 2008;104(6):063701.

[28] Miyajima Y, Adikaari AADT, Henley SJ, Shannon JM, Silva SRP.

Electrical properties of pulsed UV laser irradiated amorphous carbon. Appl Phys Lett. 2008;92(15):152104. 
[29] Miyajima Y, Shannon JM, Henley SJ, Stolejan V, Cox DC, Silva SRP. Electrical conduction mechanism in laser deposited amorphous carbon. Thin Solid Films. 2007;516(2-4):257-61.

[30] Silva SRP, Stolojan V. Electron energy loss spectroscopy of carbonaceous rnaterials. Thin Solid Films. 2005;488(1-2):283-90.

[31] Giusca CE, Tison Y, Silva SRP. Inter-Layer Interaction in Double-Walled Carbon Nanotubes Evidenced by Scanning Tunneling Microscopy and Spectroscopy. Nano. 2008;3(2):65-73.

[32] O'Leary SK. An analytical density of states and joint density of states analysis of amorphous semiconductors. J Appl Phys. 2004;96(7):3680-6.

[33] Fanchini G, Tagliaferro A. A new interpretation of the Urbach energy in amorphous carbon films. Diam Relat Mater. 2004;13(4-8):1402-7.

[34] Fanchini G, Tagliaferro A. Disorder and Urbach energy in hydrogenated amorphous carbon: A phenomenological model. Appl Phys Lett. 2004;85(5):730-2. [35] Miyajima Y, Henley SJ, Adamopoulos G, Stolojan V, Garcia-Caurel E, Drevillon B, et al. Pulsed laser deposited tetrahedral amorphous carbon with high sp(3) fractions and low optical bandgaps. J Appl Phys. 2009;105(7):073521.

[36] Godet C, Kleider JP. Disorder and localization in bandtail hopping transport: experiments and concepts. J Mater Sci-Mater El. 2006;17(6):413-26.

[37] Godet C. Physics of bandtail hopping in disordered carbons. Diam Relat Mater. 2003;12(2):159-65.

[38] Horcas I, Fernandez R, Gomez-Rodriguez JM, Colchero J, Gomez-Herrero J, Baro AM. WSXM: A software for scanning probe microscopy and a tool for nanotechnology. Review of Scientific Instruments. 2007;78(1):013705.

[39] Amaratunga GAJ, Silva SRP. Nitrogen containing hydrogenated amorphous carbon for thin-film field emission cathodes. Appl Phys Lett. 1996;68(18):2529-31.

[40] Tauc J, Grigorov.R, Vancu A. Optical Properties and Electronic Structure of Amorphous Germanium. Physica Status Solidi. 1966;15(2):627.

[41] Teo KBK, Ferrari AC, Fanchini G, Rodil SE, Yuan J, Tsai JTH, et al. Highest optical gap tetrahedral amorphous carbon. Diam Relat Mater. 2002;11(36):1086-90.

[42] Schwan J, Ulrich S, Theel T, Roth H, Ehrhardt H, Becker P, et al. Stressinduced formation of high-density amorphous carbon thin films. J Appl Phys. 1997;82(12):6024-30.

[43] Jackson WB, Amer NM, Boccara AC, Fournier D. Photothermal Deflection Spectroscopy and Detection. Applied Optics. 1981;20(8):1333-44.

[44] Fournier D, Boccara AC, Amer NM, Gerlach R. Sensitive Insitu Trace-Gas Detection by Photothermal Deflection Spectroscopy. Appl Phys Lett. 1980;37(6):519-21.

[45] Rodil S, Morrison NA, Milne WI, Robertson J, Stolojan V, Jayawardane DN. Deposition of carbon nitride films using an electron cyclotron wave resonance plasma source. Diam Relat Mater. 2000;9(3-6):524-9.

[46] Waidmann S, Knupfer M, Fink J, Kleinsorge B, Robertson J. Electronic structure studies of undoped and nitrogen-doped tetrahedral amorphous carbon using high-resolution electron energy-loss spectroscopy. J Appl Phys.

2001;89(7):3783-92. 
[47] Silva SRP, Rafferty B, Amaratunga GAJ, Schwan J, Franceschini DF, Brown LM. Nitrogenated amorphous carbon as a semiconductor. Diam Relat Mater. 1996;5(3-5):401-4.

[48] Hayashi Y, Kamada N, Soga T, Jimbo T. Characterization of amorphous carbon nitride by bottom-gated thin-film structure. Diam Relat Mater. 2006;15(48):1015-8.

[49] Ilie A, Hart A, Flewitt AJ, Robertson J, Milne WI. Effect of work function and surface microstructure on field emission of tetrahedral amorphous carbon. $\mathbf{J}$ Appl Phys. 2000;88(10):6002-10. 